\title{
Decomposition of M/M/1 With Unreliable Service and a Working Vacation
}

\author{
Joshua Patterson ${ }^{1} \&$ Andrzej Korzeniowski ${ }^{2}$ \\ ${ }^{1}$ Department of Mathematics, Texas A\&M University-Commerce, Commerce TX, USA \\ ${ }^{2}$ Department of Mathematics, University of Texas at Arlington, Arlington TX, USA \\ Correspondence: Joshua Patterson, Department of Mathematics, Texas A\&M University-Commerce, Commerce TX, \\ 75428, USA. E-mail: Joshua.Patterson@tamuc.edu
}

Received: December 4, 2019 Accepted: December 27, 2019 Online Published: January 2, 2020

doi:10.5539/ijsp.v9n1p63

URL: https://doi.org/10.5539/ijsp.v9n1p63

\begin{abstract}
We use the stationary distribution for the $\mathrm{M} / \mathrm{M} / 1$ with Unreliable Service and a Working Vacation $(\mathrm{M} / \mathrm{M} / 1 / \mathrm{US} / \mathrm{WV}$ ) given explicitly in (Patterson \& Korzeniowski, 2019) to find a decomposition of the stationary queue length N. By applying the distributional form of Little's Law the Laplace-tieltjes Transform of the stationary customer waiting time W is derived. The closed form of the expected value and variance for both $\mathrm{N}$ and $\mathrm{W}$ is found and the relationship of the expected stationary waiting time as a function of the service failure rate is determined.
\end{abstract}

Keywords: unreliable service, quasi birth death process, matrix-geometric, working vacation, decomposition

\section{Introduction}

Within the literature, the $\mathrm{M} / \mathrm{M} / 1 / \mathrm{WV}$ model comes as a generalization of the $\mathrm{M} / \mathrm{M} / 1 / \mathrm{V}$ model. The reader may see (Levy \& Yechiali, 1975) for an example of the $\mathrm{M} / \mathrm{M} / 1 / \mathrm{V}$ model within the $\mathrm{M} / \mathrm{G} / 1$ framework, and (Xu \& Tian, 2009) for an $\mathrm{M} / \mathrm{M} / 1 / \mathrm{WV}$ model within the matrix-geometric framework of Neuts (Neuts, 1981). In general, the $\mathrm{M} / \mathrm{M} / 1 / \mathrm{WV}$ model is well known and has been studied extensively.

In 2018, a new queue type was introduced: M/M/1/US (Patterson \& Korzeniowski, 2018). While unrelated to queues with a working vacation, this concept of 'Unreliable Service' was later extended to the case of the M/M/1/US/WV (Patterson \& Korzeniowski, 2019). Explicit, closed form expressions were given for the stationary queue length $\mathrm{N}$ of this M/M/1/US/WV queue. In this paper, we use the 2019 results of Patterson and Korzeniowski and obtain the following:

- P.G.F. (Probability Generating Function) of N.

- Decomposition of $\mathrm{N}$ into the sum of independent generalized random variables.

- L.S.T. (Laplace-tieltjes Transform) of the stationary customer waiting time W.

- Explicit analytical results such as $E($.$) and \operatorname{Var}($.$) for both \mathrm{N}$ and $W$.

- The relationship of the expected stationary waiting time as a function of the service failure rate.

To motivate the construction and analysis of the $\mathrm{M} / \mathrm{M} / 1 / \mathrm{US} / \mathrm{WV}$, we recall:

"A queue with unreliable service is a queue where service may be unsuccessful any number of times before it is successful. This type of queue is important to study because it occurs naturally within a lot of systems. For example, imagine trying to have a conversation with someone in a quiet environment, such as a library-words spoken are generally heard and understood (i.e. service is rendered successfully every time). Now, imagine trying to have the same conversation in a noisy environment, such as a busy restaurant-it can be done, but you may need to repeat yourself (i.e. service may fail)." (Patterson \& Korzeniowski, 2019)

The phenomenon 'repeat yourself in a noisy environment' also appears in wireless network environments, where wireless network equipment typically utilize multiple interface bit-rates that are negotiated dynamically depending on various factors such as signal strength, noise, and overall link quality. One may envision these distinct bit-rates as varying degrees of a 'working vacation,' whereby the service rate is dynamically adjusted depending on some criteria such as failure rates. In this way, we consider the $\mathrm{M} / \mathrm{M} / 1 / \mathrm{US} / \mathrm{WV}$ to be a natural extension towards the goal of modeling such wireless network queues. 


\section{Definitions}

In what follows, we define our process, state space, and the respective parameters.

Definition 2.1. Let $\{N(t) \mid t \geqslant 0\}$ be the number of customers in the queue at time $t$,

$J(t)= \begin{cases}0 & \text { the server is on a working vacation } \\ 1 & \text { the server is in a busy state }\end{cases}$

and

$S(t)= \begin{cases}1 & \text { immediately after service is rendered } \\ 0 & \text { otherwise }\end{cases}$

Then $\{(\mathrm{N}(\mathrm{t}), J(t), S(t)) \mid t \geqslant 0\}$ is a Markov process on the state space:

$\Omega=\{(0,0,0)\} \cup\{(k, j, s) \mid k \in \mathbb{N}, j, s \in\{0,1\}\}$

Define the following parameters:

- $\lambda$ : the rate of the Poisson arrivals process.

- $\mu_{\mathrm{b}}$ : the rate of service when the server is busy.

- $\mu_{v}$ : the rate of service when the server is on vacation.

- $\beta_{1}$ : the rate of a successful service determination.

- $\beta_{2}$ : the rate of a failed service determination.

- $\theta$ : vacation duration is exponentially distributed with rate $\theta$.

Definition 2.2. Vacation policy

- When the server becomes idle (i.e. $N(t)=0$ ), the server goes on a working vacation; by this we mean that customers arriving while the server is on vacation get served at a reduced rate $\mu_{v}<\mu_{\mathrm{b}}$.

- When the server is not idle (i.e. $N(t) \neq 0$ ), a vacationing server begins a working vacation duration that is exponentially distributed with rate $\theta$, after which it begins a busy period and operates at rate $\mu_{\mathrm{b}}$ until the server becomes idle again, renewing the process.

- If a customer is served successfully while the server is on a working vacation and there are additional customers waiting in the queue, the server then immediately ends its vacation and enters into a busy state until the queue is emptied.

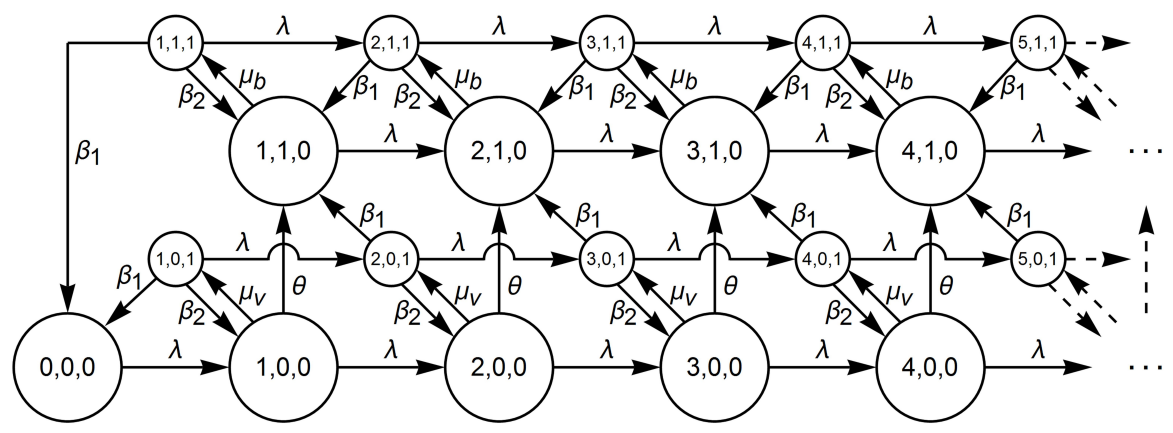

Figure 1. 3D Markovian state transition rates diagram

We define a 'successful service' to be a transition from $(n, j, 1) \longrightarrow(n-1,1,0)$ or $(n, j, 1) \longrightarrow(n-1,0,0)$, which is represented in the state transition diagram as having rate $\beta_{1}$. Accordingly, we define a 'failed service' to be a transition from $(\mathrm{n}, \mathrm{j}, 1) \longrightarrow(\mathrm{n}, \mathrm{j}, 0)$ with transition rate $\beta_{2}$.

Definition 2.3. Lexicographical Ordering

We say $\left(k_{1}, j_{1}, s_{1}\right)<\left(k_{2}, j_{2}, s_{2}\right)$ if and only if $k_{1} \frown j_{1} \frown s_{1}<k_{2} \frown j_{2} \frown s_{2}$,

where $\frown$ denotes concatenation (Quine, 1946). For example, $7 \frown 0 \frown 1=701$.

Using this ordering convention we can write:

$\Omega=\{(0,0,0),(1,0,0),(1,0,1),(1,1,0),(1,1,1), \ldots\}$ and define the corresponding infinitesimal matrix $\mathrm{Q}$. 


\subsection{Infinitesimal Matrix $Q$}

$$
\mathbf{Q}=\left[\begin{array}{cccccc}
\hat{\mathbf{A}} & \hat{\mathbf{C}} & 0 & 0 & 0 & \ldots \\
\hat{\mathbf{B}} & \mathbf{A} & \mathbf{C} & 0 & 0 & \ldots \\
0 & \mathbf{B} & \mathbf{A} & \mathbf{C} & 0 & \ldots \\
\vdots & \vdots & \vdots & \ddots & \vdots & \vdots
\end{array}\right]
$$

where

$$
\begin{aligned}
& \hat{\mathbf{A}}=[-\lambda] \quad \hat{\mathbf{B}}=\left[\begin{array}{c}
0 \\
\beta_{1} \\
0 \\
\beta_{1}
\end{array}\right] \\
& \mathbf{A}=\left[\begin{array}{cccc}
-\left(\lambda+\mu_{v}+\theta\right) & \mu_{v} & \theta & 0 \\
\beta_{2} & -\left(\lambda+\beta_{1}+\beta_{2}\right) & 0 & 0 \\
0 & 0 & -\left(\lambda+\mu_{\mathrm{b}}\right) & \mu_{\mathrm{b}} \\
0 & 0 & \beta_{2} & -\left(\lambda+\beta_{1}+\beta_{2}\right)
\end{array}\right] \\
& \begin{aligned}
\mathbf{C} & =\left[\begin{array}{llll}
\lambda & 0 & 0 & 0 \\
0 & \lambda & 0 & 0 \\
0 & 0 & \lambda & 0 \\
0 & 0 & 0 & \lambda
\end{array}\right] \\
\mathbf{B} & =\left[\begin{array}{llcc}
0 & 0 & 0 & 0 \\
0 & 0 & \beta_{1} & 0 \\
0 & 0 & 0 & 0 \\
0 & 0 & \beta_{1} & 0
\end{array}\right]
\end{aligned}
\end{aligned}
$$

\subsection{The Matrix $R$}

Let $\mathbf{R}$ be the solution to the quadratic matrix equation:

$$
\mathbf{R}^{2} \mathbf{B}+\mathbf{R A}+\mathbf{C}=\mathbf{0}
$$

$\underline{\text { Remark }} \mathbf{R}$ is given explicitly in (Patterson \& Korzeniowski, 2019).

Let $\left\{\rho_{i}\right\}_{i=0,1,2,3}$ be the eigenvalues of $\mathbf{R}$ such that $\rho_{0}=\operatorname{sp}(\mathbf{R})$, then $\{(\mathrm{N}(\mathrm{t}), \mathrm{J}(\mathrm{t}), \mathrm{S}(\mathrm{t})) \mid t \geqslant 0\}$ is positive recurrent if and only if $\rho_{0}<1$ (Patterson \& Korzeniowski, 2019). Lastly, we define $K=\left(1-\rho_{0}\right)\left(1-\rho_{1}\right)$.

\section{Decomposition of $\mathbf{N}$}

Theorem 3.1. If $\mathrm{K}>0$, then the stationary number of customers in steady-state, $\mathrm{N}$, can be decomposed into the sum of four independent geometric random variables and an independent finitely valued generalized random variable with an explicitly known generalized distribution. Namely: $\mathrm{N}=\mathrm{X}_{0}+\mathrm{X}_{1}+\mathrm{X}_{2}+\mathrm{X}_{3}+\mathrm{X}_{4}$, where:

$X_{0} \sim \operatorname{Geometric}\left(1-\rho_{0}\right), X_{1} \sim \operatorname{Geometric}\left(1-\rho_{1}\right), X_{2} \sim \operatorname{Geometric}\left(1-\rho_{2}\right), X_{3} \sim \operatorname{Geometric}\left(1-\rho_{3}\right)$ and

$$
\mathrm{P}\left(\mathrm{X}_{4}=\mathrm{k}\right)= \begin{cases}\mathrm{K}^{*} \widehat{\delta_{0}} & \text { if } \mathrm{k}=0 \\ \mathrm{~K}^{*} \widehat{\delta_{1}} & \text { if } \mathrm{k}=1 \\ \mathrm{~K}^{*} \widehat{\delta_{2}} & \text { if } \mathrm{k}=2 \\ 0 & \text { otherwise }\end{cases}
$$

with $\mathrm{K}^{*}$ and $\left\{\widehat{\delta_{i}}\right\}_{i=0}^{2}$ defined as follows:

$$
\begin{aligned}
K^{*} & =\frac{K}{\left(1-\rho_{0}\right)\left(1-\rho_{1}\right)\left(1-\rho_{2}\right)\left(1-\rho_{3}\right)} \\
& =\frac{\mu_{\mathrm{b}}\left((\theta+\lambda) \beta_{2}+\lambda\left(\theta+\lambda+\mu_{v}\right)+\beta_{1}\left(\theta+\lambda+\mu_{v}\right)\right)}{(\theta+\lambda)\left(\beta_{1}+\beta_{2}\right) \mu_{\mathrm{b}}+\left(\left(\beta_{1}\left(\mu_{\mathrm{b}}-\lambda\right)-\lambda\left(\beta_{2}+\mu_{\mathrm{b}}\right)\right)+\lambda \mu_{\mathrm{b}}\right) \mu_{v}} \\
& \left\{\begin{array}{l}
\widehat{\delta_{0}}=1 \\
\widehat{\delta_{1}}=-\frac{\lambda\left(\left(\lambda+\beta_{1}+\beta_{2}\right) \mu_{v}+\mu_{\mathrm{b}}\left(\theta+\lambda+\mu_{v}\right)\right)}{\mu_{\mathrm{b}}\left((\theta+\lambda) \beta_{2}+\lambda\left(\theta+\lambda+\mu_{v}\right)+\beta_{1}\left(\theta+\lambda+\mu_{v}\right)\right)} \\
\widehat{\delta_{2}}=\frac{\lambda^{2} \mu_{v}}{\mu_{\mathrm{b}}\left((\theta+\lambda) \beta_{2}+\lambda\left(\theta+\lambda+\mu_{v}\right)+\beta_{1}\left(\theta+\lambda+\mu_{v}\right)\right)}
\end{array}\right.
\end{aligned}
$$

Proof. Since all exponential rates $\lambda, \mu_{\mathrm{b}}, \mu_{v}, \beta_{1}, \beta_{2}, \theta$ are non-negative, then by inspection we have:

$\mathrm{K}^{*}>0, \widehat{\delta_{0}}>0, \widehat{\delta_{1}}<0$, and $\widehat{\delta_{2}}>0$.

We also note that

$$
\left(1-\rho_{0}\right)\left(1-\rho_{1}\right)=\frac{\beta_{1}\left(\mu_{\mathfrak{b}}-\lambda\right)-\lambda\left(\mu_{\mathfrak{b}}+\beta_{2}\right)}{\beta_{1} \mu_{\mathrm{b}}} \text {, and }\left(1-\rho_{2}\right)\left(1-\rho_{3}\right)=\frac{\theta \beta_{2}+\beta_{1}\left(\theta+\mu_{v}\right)}{(\theta+\lambda) \beta_{2}+\lambda\left(\theta+\lambda+\mu_{v}\right)+\beta_{1}\left(\theta+\lambda+\mu_{v}\right)}
$$

These can be verified from the definitions given in (Patterson \& Korzeniowski, 2019). 
Therefore, using the definition of $\mathrm{K}$, we have

$$
\begin{aligned}
K^{*} & =\frac{K}{\left(1-\rho_{0}\right)\left(1-\rho_{1}\right)\left(1-\rho_{2}\right)\left(1-\rho_{3}\right)} \\
& =\frac{\left(\beta_{1}\left(\mu_{\mathrm{b}}-\lambda\right)-\lambda\left(\mu_{\mathrm{b}}+\beta_{2}\right)\right)\left(\theta \beta_{2}+\beta_{1}\left(\theta+\mu_{v}\right)\right)}{\beta_{1}\left((\theta+\lambda)\left(\beta_{1}+\beta_{2}\right) \mu_{\mathrm{b}}+\left(\left(\beta_{1}\left(\mu_{\mathrm{b}}-\lambda\right)-\lambda\left(\mu_{\mathrm{b}}+\beta_{2}\right)\right)+\lambda \mu_{\mathrm{b}}\right) \mu_{v}\right)} \frac{1}{\left(1-\rho_{0}\right)\left(1-\rho_{1}\right)\left(1-\rho_{2}\right)\left(1-\rho_{3}\right)} \\
& =\frac{\mu_{\mathrm{b}}\left((\theta+\lambda) \beta_{2}+\lambda\left(\theta+\lambda+\mu_{v}\right)+\beta_{1}\left(\theta+\lambda+\mu_{v}\right)\right)}{\left((\theta+\lambda)\left(\beta_{1}+\beta_{2}\right) \mu_{\mathrm{b}}+\left(\left(\beta_{1}\left(\mu_{\mathrm{b}}-\lambda\right)-\lambda\left(\beta_{2}+\mu_{\mathrm{b}}\right)\right)+\lambda \mu_{\mathrm{b}}\right) \mu_{v}\right)}
\end{aligned}
$$

By definition the Probability Generating Function of $N$ is given by

$$
\begin{aligned}
& \mathcal{G}_{\mathcal{N}}(z)=\sum_{k=0}^{\infty} \mathrm{P}(\mathrm{N}=k) z^{k} \\
& \Longrightarrow \mathcal{G}_{\mathcal{N}}(z)=\sum_{k=0}^{\infty} \mathrm{P}(\mathrm{N}=\mathrm{k}) z^{\mathrm{k}} \\
& =\pi_{00}+\sum_{k=1}^{\infty}\left(\pi_{k 0}+\pi_{k 1}+\pi_{k 2}+\pi_{k 3}\right) z^{k} \\
& =\sum_{k=0}^{\infty}\left(\pi_{k 0}+\pi_{k 1}+\pi_{k 2}+\pi_{k 3}\right) z^{k} \\
& \vdots \\
& =\frac{K^{*}\left(1-\rho_{0}\right)\left(1-\rho_{1}\right)\left(1-\rho_{2}\right)\left(1-\rho_{3}\right)}{\left(1-\rho_{0} z\right)\left(1-\rho_{1} z\right)\left(1-\rho_{2} z\right)\left(1-\rho_{3} z\right)}\left(\widehat{\delta_{0}}+\widehat{\delta_{1}} z+\widehat{\delta_{2}} z^{2}\right) \\
& =\frac{1-\rho_{0}}{1-\rho_{0} z} \frac{1-\rho_{1}}{1-\rho_{1} z} \frac{1-\rho_{2}}{1-\rho_{2} z} \frac{1-\rho_{3}}{1-\rho_{3} z} K^{*}\left(\widehat{\delta_{0}}+\widehat{\delta_{1}} z+\widehat{\delta_{2}} z^{2}\right) \\
& =\mathcal{G}_{X_{0}}(z) \mathcal{G}_{X_{1}}(z) \mathcal{G}_{X_{2}}(z) \mathcal{G}_{X_{3}}(z) K^{*}\left(\widehat{\delta_{0}}+\widehat{\delta_{1}} z+\widehat{\delta_{2}} z^{2}\right)
\end{aligned}
$$

Since $\mathcal{G}_{\mathcal{N}}(1)=\mathcal{G}_{X_{0}}(1) \mathcal{G}_{X_{1}}(1) \mathcal{G}_{X_{2}}(1) \mathcal{G}_{X_{3}}(1) K^{*}\left(\widehat{\delta_{1}}+\widehat{\delta_{2}}+\widehat{\delta_{3}}\right)$ and since all rates are finite

$\Longrightarrow \mathrm{K}^{*}\left(\widehat{\delta_{1}}+\widehat{\delta_{2}} z+\widehat{\delta_{3}} z^{2}\right)=1$ when $z=1$, and $\sum_{i=1}^{3}\left|\widehat{\delta}_{i}\right|<\infty$.

$\Longrightarrow \mathcal{G N}_{N}(z)=\mathcal{G X}_{x_{0}}(z) \mathcal{G}_{x_{1}}(z) \mathcal{G}_{X_{2}}(z) \mathcal{G}_{x_{3}}(z) \mathcal{G}_{X_{4}}(z)$, where $\mathcal{G}_{x_{4}}(z)$ is said to be the generalized generating function for the generalized random variable $X_{4}$.

Remark. For reasons of length we omit the rather lengthy algebraic steps represented by the vertical dots that reduced the infinite series to its simplest form. It is worth noting that for verification purposes, the algebraic capabilities of Wolfram's Mathematica were utilized as well.

Thanks to The Fundamental Theorem of Negative Probabilities by Ruzsa, Imre and Székely, Gábor J. (Ruzsa \& Székely, 1988), we are guaranteed the existence of a pair of ordinary random variables $Y_{0}, Y_{1}$ such that $Y_{1}=Y_{0}+X_{4}$ in distribution. Indeed, in our case we see that $Y_{1}=N$ and $Y_{0}=X_{0}+X_{1}+X_{2}+X_{3}$ is such a pair.

\section{The L.S.T. of W}

Proposition 4.1. Using the distributional form of Little's Law (Little, 1961), proven in the general case by Keilson and Servi (Keilson $\mathcal{E}$ Servi LD, 1988), the Laplace-tieltjes Transform of the stationary waiting time W for the queue with the stationary number of customers $\mathrm{N}$ is given by

$$
\mathcal{W}^{*}(s)=\frac{\frac{\lambda\left(1-\rho_{0}\right)}{\rho_{0}}}{\left(\frac{\lambda\left(1-\rho_{0}\right)}{\rho_{0}}-s\right)} \frac{\frac{\lambda\left(1-\rho_{1}\right)}{\rho_{1}}}{\left(\frac{\lambda\left(1-\rho_{1}\right)}{\rho_{1}}-s\right)} \frac{\frac{\lambda\left(1-\rho_{2}\right)}{\rho_{2}}}{\left(\frac{\lambda\left(1-\rho_{2}\right)}{\rho_{2}}-s\right)} \frac{\frac{\lambda\left(1-\rho_{3}\right)}{\rho_{3}}}{\left(\frac{\lambda\left(1-\rho_{3}\right)}{\rho_{3}}-s\right)} K^{*}\left(\widehat{\delta_{0}}+\widehat{\delta_{1}}+\widehat{\delta_{2}}-\left(\frac{\widehat{\delta_{1}}}{\lambda}+\frac{2 \widehat{\delta_{2}}}{\lambda}\right) s+\frac{\widehat{\delta_{2}}}{\lambda^{2}} s^{2}\right)
$$

Proof.

$$
\begin{aligned}
\mathcal{W}^{*}(s)=\mathcal{G}_{N}\left(1-\frac{s}{\lambda}\right) & =\frac{K^{*}\left(1-\rho_{0}\right)\left(1-\rho_{1}\right)\left(1-\rho_{2}\right)\left(1-\rho_{3}\right)\left(\widehat{\delta_{0}}+\widehat{\delta_{1}}\left(1-\frac{s}{\lambda}\right)+\widehat{\delta_{2}}\left(1-\frac{s}{\lambda}\right)^{2}\right)}{\left(1-\rho_{0}\left(1-\frac{s}{\lambda}\right)\right)\left(1-\rho_{1}\left(1-\frac{s}{\lambda}\right)\right)\left(1-\rho_{2}\left(1-\frac{s}{\lambda}\right)\right)\left(1-\rho_{3}\left(1-\frac{s}{\lambda}\right)\right)} \\
& =\frac{K^{*}\left(1-\rho_{0}\right)\left(1-\rho_{1}\right)\left(1-\rho_{2}\right)\left(1-\rho_{3}\right)\left(\widehat{\delta_{0}}+\widehat{\delta_{1}}\left(1-\frac{s}{\lambda}\right)+\widehat{\delta_{2}}\left(1-\frac{s}{\lambda}\right)^{2}\right)}{\left(1-\rho_{0}\left(1-\frac{s}{\lambda}\right)\right)\left(1-\rho_{1}\left(1-\frac{s}{\lambda}\right)\right)\left(1-\rho_{2}\left(1-\frac{s}{\lambda}\right)\right)\left(1-\rho_{3}\left(1-\frac{s}{\lambda}\right)\right)} \\
& =\frac{K^{*} \lambda^{4}\left(1-\rho_{0}\right)\left(1-\rho_{1}\right)\left(1-\rho_{2}\right)\left(1-\rho_{3}\right)\left(\widehat{\delta_{0}}+\widehat{\delta_{1}}\left(1-\frac{s}{\lambda}\right)+\widehat{\delta_{2}}\left(1-\frac{s}{\lambda}\right)^{2}\right)}{\left(\lambda-\rho_{0}(\lambda-s)\right)\left(\lambda-\rho_{1}(\lambda-s)\right)\left(\lambda-\rho_{2}(\lambda-s)\right)\left(\lambda-\rho_{3}(\lambda-s)\right)}
\end{aligned}
$$




$$
\begin{aligned}
& =\frac{\mathrm{K}^{*} \lambda^{4}\left(1-\rho_{0}\right)\left(1-\rho_{1}\right)\left(1-\rho_{2}\right)\left(1-\rho_{3}\right)\left(\widehat{\delta_{0}}+\widehat{\delta_{1}}\left(1-\frac{s}{\lambda}\right)+\widehat{\delta_{2}}\left(1-\frac{s}{\lambda}\right)^{2}\right)}{\left(\lambda\left(1-\rho_{0}\right)-\rho_{0} s\right)\left(\lambda\left(1-\rho_{1}\right)-\rho_{1} s\right)\left(\lambda\left(1-\rho_{2}\right)-\rho_{2} s\right)\left(\lambda\left(1-\rho_{3}\right)-\rho_{3} s\right)} \\
& =\frac{\frac{\lambda\left(1-\rho_{0}\right)}{\rho_{0}} \frac{\lambda\left(1-\rho_{1}\right)}{\rho_{1}} \frac{\lambda\left(1-\rho_{2}\right)}{\rho_{2}} \frac{\lambda\left(1-\rho_{3}\right)}{\rho_{3}} K^{*}\left(\widehat{\delta_{0}}+\widehat{\delta_{1}}\left(1-\frac{s}{\lambda}\right)+\widehat{\delta_{2}}\left(1-\frac{s}{\lambda}\right)^{2}\right)}{\left(\frac{\lambda\left(1-\rho_{0}\right)}{\rho_{0}}-s\right)\left(\frac{\lambda\left(1-\rho_{1}\right)}{\rho_{1}}-s\right)\left(\frac{\lambda\left(1-\rho_{2}\right)}{\rho_{2}}-s\right)\left(\frac{\lambda\left(1-\rho_{3}\right)}{\rho_{3}}-s\right)} \\
& =\frac{\frac{\lambda\left(1-\rho_{0}\right)}{\rho_{0}}}{\left(\frac{\lambda\left(1-\rho_{0}\right)}{\rho_{0}}-s\right)} \frac{\frac{\lambda\left(1-\rho_{1}\right)}{\rho_{1}}}{\left(\frac{\lambda\left(1-\rho_{1}\right)}{\rho_{1}}-s\right)} \frac{\frac{\lambda\left(1-\rho_{2}\right)}{\rho_{2}}}{\left(\frac{\lambda\left(1-\rho_{2}\right)}{\rho_{2}}-s\right)} \frac{\frac{\lambda\left(1-\rho_{3}\right)}{\rho_{3}}}{\left(\frac{\lambda\left(1-\rho_{3}\right)}{\rho_{3}}-s\right)} K^{*}\left(\widehat{\delta_{0}}+\widehat{\delta_{1}}+\widehat{\delta_{2}}-\left(\frac{\widehat{\delta_{1}}}{\lambda}+\frac{2 \widehat{\delta_{2}}}{\lambda}\right) s+\frac{\widehat{\delta_{2}}}{\lambda^{2}} s^{2}\right)
\end{aligned}
$$

\section{Results}

\subsection{Analytical Results}

Table 1. Analytical Results on N

\begin{tabular}{ll}
\hline & $\mathcal{G}_{\mathrm{N}}(z)=\frac{1-\rho_{0}}{1-\rho_{0} z} \frac{1-\rho_{1}}{1-\rho_{1} z} \frac{1-\rho_{2}}{1-\rho_{2} z} \frac{1-\rho_{3}}{1-\rho_{3} z} K^{*}\left(\widehat{\delta_{0}}+\widehat{\delta_{1}} z+\widehat{\delta_{2}} z^{2}\right)$ \\
\hline $\mathrm{E}()$. & $\frac{\rho_{0}}{1-\rho_{0}}+\frac{\rho_{1}}{1-\rho_{1}}+\frac{\rho_{2}}{1-\rho_{2}}+\frac{\rho_{3}}{1-\rho_{3}}+\mathrm{K}^{*}\left(\widehat{\rho_{1}}+2 \widehat{\rho_{2}}\right)$ \\
$\operatorname{Var}()$. & $\frac{\rho_{0}}{\left(1-\rho_{0}\right)^{2}}+\frac{\rho_{2}}{\left(1-\rho_{1}\right)^{2}}+\frac{\rho_{2}}{\left(1-\rho_{2}\right)^{2}}+\frac{\rho^{2}}{\left(1-\rho_{3}\right)^{2}}-\mathrm{K}^{*} \widehat{\delta_{1}}\left(3+\mathrm{K}^{*} \widehat{\delta_{1}}\right)$ \\
\hline
\end{tabular}

Table 2. Analytical Results on W

$$
\begin{array}{ll}
\mathcal{W}^{*}(s)=\frac{\frac{\lambda\left(1-\rho_{0}\right)}{\rho_{0}}}{\left(\frac{\lambda\left(1-\rho_{0}\right)}{\rho_{0}}-s\right)} \frac{\frac{\lambda\left(1-\rho_{1}\right)}{\rho_{1}}}{\left(\frac{\lambda\left(1-\rho_{1}\right)}{\rho_{1}}-s\right)} \frac{\frac{\lambda\left(1-\rho_{2}\right)}{\rho_{2}}}{\left(\frac{\lambda\left(1-\rho_{2}\right)}{\rho_{2}}-s\right)} \frac{\frac{\lambda\left(1-\rho_{3}\right)}{\rho_{3}}}{\left(\frac{\lambda\left(1-\rho_{3}\right)}{\rho_{3}}-s\right)} K^{*}\left(\widehat{\delta_{0}}+\widehat{\delta_{1}}+\widehat{\delta_{2}}-\left(\frac{\rho_{1}}{\lambda}+\frac{2 \widehat{\delta_{2}}}{\lambda}\right) s+\frac{\widehat{\delta_{2}}}{\lambda^{2}} s^{2}\right) \\
\mathrm{E}(.) \quad \frac{\rho_{0}}{\lambda\left(1-\rho_{0}\right)}+\frac{\rho_{1}}{\lambda\left(1-\rho_{1}\right)}+\frac{\rho_{2}}{\lambda\left(1-\rho_{2}\right)}+\frac{\rho_{3}}{\lambda\left(1-\rho_{3}\right)}+\frac{K^{*}}{\lambda}\left(\widehat{\delta_{1}}+2 \widehat{\delta_{2}}\right) \\
\left.\operatorname{Var}(.) \quad \frac{\rho_{0}^{2}}{\lambda^{2}\left(1-\rho_{0}\right)^{2}}+\frac{\rho_{1}^{2}}{\lambda^{2}\left(1-\rho_{1}\right)^{2}}+\frac{\rho_{2}^{2}}{\lambda^{2}\left(1-\rho_{2}\right)^{2}}+\frac{\rho_{3}^{2}}{\lambda^{2}\left(1-\rho_{3}\right)^{2}}+\frac{\rho^{2}}{\lambda^{2}}-K^{*}\left(\widehat{\delta_{1}}+2 \widehat{\delta_{2}}\right)^{2}\right)
\end{array}
$$

\subsection{Numerical Results}

When choosing parameters $\theta, \beta_{1}, \beta_{2}, \mu_{v}, \mu_{\mathrm{b}}, \lambda$ to illustrate as an example, it follows that one might seek to make $\rho_{0}, \rho_{1}, \rho_{2}$, and $\rho_{3}$ given in (Patterson \& Korzeniowski, 2019) rational. However, we show that if one is interested in $E(N)$ or $E(W)$, then this in not necessary with the following Lemma.

Lemma 5.1. For any $\theta, \beta_{1}, \beta_{2}, \mu_{v}, \mu_{\mathrm{b}}, \lambda \in \mathbb{Q}$, we have $\mathrm{E}(\mathrm{N}) \in \mathbb{Q}$ and $\mathrm{E}(\mathrm{W}) \in \mathbb{Q}$.

Proof. By Table 1, we have

$$
\begin{aligned}
E(N) & =\frac{\rho_{0}}{1-\rho_{0}}+\frac{\rho_{1}}{1-\rho_{1}}+\frac{\rho_{2}}{1-\rho_{2}}+\frac{\rho_{3}}{1-\rho_{3}}+K^{*}\left(\widehat{\delta_{1}}+2 \widehat{\delta_{2}}\right) \\
& =\frac{\rho_{0}\left(1-\rho_{1}\right)+\rho_{1}\left(1-\rho_{0}\right)}{\left(1-\rho_{1}\right)\left(1-\rho_{0}\right)}+\frac{\rho_{2}\left(1-\rho_{3}\right)+\rho_{3}\left(1-\rho_{2}\right)}{\left(1-\rho_{2}\right)\left(1-\rho_{3}\right)}+K^{*}\left(\widehat{\delta_{1}}+2 \widehat{\delta_{2}}\right) \\
& =\frac{\rho_{0}+\rho_{1}-2 \rho_{0} \rho_{1}}{\left(1-\rho_{1}\right)\left(1-\rho_{0}\right)}+\frac{\rho_{2}+\rho_{3}-2 \rho_{2} \rho_{3}}{\left(1-\rho_{2}\right)\left(1-\rho_{3}\right)}+K^{*}\left(\widehat{\delta_{1}}+2 \widehat{\delta_{2}}\right), \text { where }
\end{aligned}
$$




$$
\begin{array}{ll}
\rho_{0} \rho_{1}=\frac{\lambda^{2}}{\mu_{\mathrm{b}} \beta_{1}} & \rho_{0}+\rho_{1}=\frac{\lambda\left(\beta_{1}+\beta_{2}+\lambda+\mu_{\mathrm{b}}\right)}{\mu_{\mathrm{b}} \beta_{1}} \\
\rho_{2} \rho_{3}=\frac{\lambda^{2}}{\beta_{2}(\theta+\lambda)+\beta_{1}\left(\theta+\lambda+\mu_{v}\right)+\lambda\left(\theta+\lambda+\mu_{v}\right)} & \rho_{2}+\rho_{3}=\frac{\lambda\left(\beta_{1}+\beta_{2}+\theta+2 \lambda+\mu_{v}\right)}{\beta_{2}(\theta+\lambda)+\beta_{1}\left(\theta+\lambda+\mu_{v}\right)+\lambda\left(\theta+\lambda+\mu_{v}\right)} \\
\left(1-\rho_{0}\right)\left(1-\rho_{1}\right)=\frac{\beta_{1}\left(\mu_{\mathrm{b}}-\lambda\right)-\lambda\left(\beta_{2}+\mu_{\mathrm{b}}\right)}{\mu_{\mathrm{b}} \beta_{1}} & \left(1-\rho_{2}\right)\left(1-\rho_{3}\right)=\frac{\theta \beta_{2}+\beta_{1}\left(\theta+\mu_{v}\right)}{(\theta+\lambda) \beta_{2}+\lambda\left(\theta+\lambda+\mu_{v}\right)+\beta_{1}\left(\theta+\lambda+\mu_{v}\right)}
\end{array}
$$

The above identities can be verified from (Patterson \& Korzeniowski, 2019).

$\mathrm{K}^{*}, \widehat{\delta_{1}}$, and $\widehat{\delta_{2}}$ are rational and given in Theorem 3.1

Finally, by Little's Law (Little, 1961), we have $E(N)=\lambda E(W)$.

We chose the following values for parameters $\theta, \mu_{v}, \mu_{b}, \lambda$.

$$
\begin{cases}\lambda=10 & \theta=5 \\ \mu_{\mathrm{b}}=15 & \mu_{v}=10\end{cases}
$$

and obtain $E(W)=\frac{15 \beta_{1}^{2}+38 \beta_{1}^{3}-600 \beta_{2}-90 \beta_{1} \beta_{2}+57 \beta_{1}^{2} \beta_{2}-105 \beta_{2}^{2}+18 \beta_{1} \beta_{2}^{2}-\beta_{2}^{3}}{5\left(\beta_{1}-2 \beta_{2}-30\right)\left(3 \beta_{1}+\beta_{2}\right)\left(11 \beta_{1}+5 \beta_{2}\right)}$

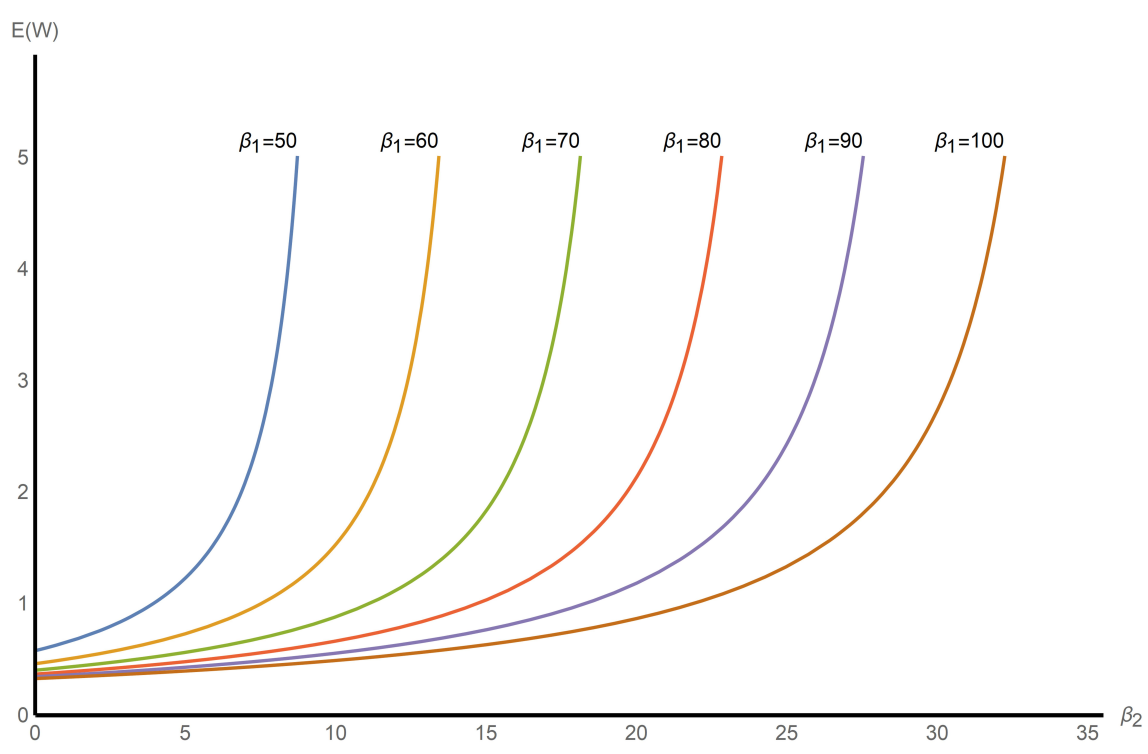

Figure 2. Plot of $E(W)$ as a function of $\beta_{2}$

The above graph shows that for a fixed success rate $\beta_{1}$, the expected waiting time increases asymptotically as $\beta_{2}$ approaches $\frac{\beta_{1}-30}{2}$. It is also worth noting that in magnitude, $\beta_{2}$ need not be large relative to $\beta_{1}$ to have a significant impact on the expected waiting time.

\section{References}

Keilson, J., \& Servi, L. D. (1988). A distributional form of Littles law. Operations Research Letters, 7(5). 223-227. https://doi.org/10.1016/0167-6377(88)90035-1

Levy, Y., \& Yechiali, U. (1975). Utilization of Idle Time in an M/G/1 Queueing System. Management Science, 22, 202-211. https://doi.org/10.1287/mnsc.22.2.202

Little, J. D. C. (1961). A Proof for the Queuing Formula: L $=\lambda W$. Operations Research Letters, 9(3), 383-387. https://doi.org/10.1287/opre.9.3.383

Neuts, M. F. (1981). Matrix-geometric Solutions in Stochastic Models: An Algorithmic Approach. Mineola, NY: Dover Publications.

Patterson, J., \& Korzeniowski, A. (2018). M/M/1 Model with Unreliable Service. International Journal of Statistics and Probability, 7(1). https://doi.org/10.5539/ijsp.v7n1p125 
Patterson, J., \& Korzeniowski, A. (2019). M/M/1 Model With Unreliable Service and a Working Vacation. International Journal of Statistics and Probability, 8(2). https://doi.org/10.5539/ijsp.v8n2p1

Ruzsa, I., \& Székely, G. (1988) Algebraic Probability Theory. John Wiley \& Sons Inc.

Xu, X., \& Tian, N. (2009). Performance Analysis of an M/M/1 Working Vacation Queue with Setup Times. Advances in Queueing Theory and Network Applications. New York, NY: Springer https://doi.org/10.1007/978-0-387-09703-9_4

Quine, W. V. (1946). Concatenation as a Basis for Arithmetic. The Journal of Symbolic Logic, 11(4), 105-114. https://projecteuclid.org/euclid.jsl/1183395170

\section{Copyrights}

Copyright for this article is retained by the author(s), with first publication rights granted to the journal.

This is an open-access article distributed under the terms and conditions of the Creative Commons Attribution license (http://creativecommons.org/licenses/by/4.0/). 